\title{
Estudio preliminar sobre patrones fonológicos según la fonología no lineal de niños chilenos de entre 3.0 y 3.11 años con Desarrollo Fonológico Prolongado (DFP)
}

\author{
A preliminary study about phonological patterns according \\ to the Non-Linear Phonology of Chilean children between \\ 3.0 and 3.11 years with Protracted Phonological \\ Development (PPD)
}

Denisse Pérez

Universidad de Valparaíso

Lirayén Delgado

Universidad de Valparaíso

Elsa Belmar

Universidad de Valparaíso

Valentina Machuca

Universidad de Valparaíso

Sandra Millapán

Universidad de Valparaíso

Ninoska Montoya

Universidad de Valparaíso

Camila Pizarro

Universidad de Valparaíso

Contacto con la autora: Denisse Pérez

Valparaíso - Chile Correo-e: denisse.perez@uv.cl

Recibido: $31 / 08 / 2018$ Aceptado: 17/04/2019

\section{RESUMEN}

El Desarrollo Fonológico Prolongado (DFP) presenta patrones de simplificación que persisten más allá de la edad esperada, disminuyendo la inteligibilidad en los niños (Dubasik \& Ingram, 2013). En el presente trabajo se describen los patrones fonológicos, según la Fonología No Lineal, en niños chilenos de entre 3.0 y 3.11 años con DFP. Este objetivo surgió de la necesidad de conocer los patrones suprasegmentales y segmentales de niños chilenos con DFP, puesto que no se ha realizado un estudio basado en la Teoría No Lineal en la población chilena. La muestra estuvo conformada por cinco niños pertenecientes a jardines infantiles de la Región de Valparaíso, Chile. A estos niños se les aplicó la Lista de Palabras del Español, obteniendo un corpus de 500 palabras. Este fue analizado a través de las medidas suprasegmentales Whole Word Match (WWM) o coincidencia de la palabra completa, Word Shape Match (WSM) o coincidencia de la estructura de la palabra, patrones acentuales; y medidas segmentales, en Porcentaje de Consonantes Correctas (PCC). Como resultado se obtuvo que los niños con DFP presentan puntajes altos en patrones acentuales (99.2\%), pero muestran una alteración en las medidas de WWM, con un 52.2\% de aciertos; WSM, con un $73.4 \%$, y PCC, con un $89.2 \%$. En consecuencia, se evidenció alteración suprasegmental y segmental en niños con DFP, pues los valores obtenidos no coinciden con el Desarrollo Típico (DT) observados en otras investigaciones.

Palabras clave: desarrollo fonológico prolongado, fonología no lineal, palabras totales coincidentes, estructura de la palabra coincidente, patrones acentuales, porcentaje de consonantes correctas.

\begin{abstract}
Protracted Phonological Development (PPD) features phonological patterns that persist beyond expected during development, decreasing intelligibility (Dubasik \& Ingram, 2013). This study aims at describing the phonological patterns in Chilean children (3.0 to 3.11 years of age) with PPD, within the Nonlinear Phonology background. This objective sprung from the need to study the suprasegmental and segmental patterns of Chilean children with PPD, given that no research has been conducted based on the Nonlinear Theory among Chilean children. The sample consisted of 5 Chilean children. They had all been diagnosed with PPD and attended kindergartens in Valparaíso, Chile. These children were administered the Spanish Word List, which allowed to obtain a corpus of 500 words. The corpus was later analyzed by means of the suprasegmental measures: i.e.: Whole Word Match (WWM), Word Shape Match (WSM) and segmental measures, expressed in Percentage of Correct Consonants (PCC). Results showed that these children with PPD performed high on accent patterns (99.2\%), but at deficit levels on the WWM measures (with $52.2 \%$ of correct answers), on the WSM measures (with 73.4\%), and on PCC measures (with 89.2\%). Consequently, this research provides empirical evidence regarding suprasegmental and segmental alterations in children with PPD, since the values herein obtained are not aligned with the Typical Development (TD) observed in other studies.
\end{abstract}

Keywords: protracted phonological development, non-linear phonology, whole word match, word shape match, stress, percentage of correct consonants. 


\section{Introducción}

La Fonología ha sido definida como la representación mental que el hablante posee de la producción y percepción de las señales del habla (Prieto, 2014). Diversas teorías han intentado explicar este componente del lenguaje. Entre ellas se encuentran: la fonología estructural (Bloomfield, 1984; Harris, 1951; Jones, 1957), la fonología generativa (Chomsky \& Halle, 1979), la fonología natural (Ingram, 1983; Stampe, 1969), la fonología generativa natural (Hooper, 1976; Vennemann, 1971), la fonología no lineal (Clements, 1985; Goldsmith, 1976) y la fonología de la optimidad (Kager, 1990; McCarthy \& Prince, 1993).

La presente investigación se fundamenta en la fonología no lineal, la cual transita desde de un paradigma lineal a uno tridimensional o por capas, abordando factores antes no considerados íntegramente (Rello, 2007). Algunos de ellos son: la frase, la estructura de la palabra, los segmentos (fonemas) y los rasgos, los cuales se relacionan entre sí por líneas de asociación y se organizan jerárquicamente (M. Bernhardt \& Zhao, 2010; Lloret, 1992).

Entre los modelos que forman parte de la fonología no lineal destacan: la fonología métrica o prosódica (Liberman, 1975; Liberman \& Prince, 1977); la fonología léxica, propuesta por Kiparsky (1982) y (Mohanan, 1982); y la fonología autosegmental fundada por (Goldsmith, 1976). La fonología no lineal ha permitido incorporar nuevos enfoques al análisis fonológico infantil en las unidades suprasegmentales y las unidades segmentales. Dentro de las unidades suprasegmentales se encuentran: (1) Coincidencia
Palabra Completa, en inglés Whole Word Match (WWM), que se refiere a la proporción de palabras producidas por un niño que coinciden con el modelo adulto (Bernhardt et al., 2015), (2) Coincidencia de Estructura de la Palabra o Word Shape Match (WSM) en inglés, parámetro que mide la proporción de palabras producidas por el niño que, según la estructura silábica, coinciden con el modelo adulto (Bernhardt et al., 2015) y (3) acento prosódico, rasgo fundamental que se puede definir como el mayor grado de prominencia de una sílaba con respecto a las otras dentro de una misma palabra (Real Academia Española, 2011). Por su parte dentro de las unidades segmentales se encuentra el porcentaje de consonantes correctas (PCC) producidas por los menores (Shriberg \& Kwiatkowski, 1982).

Sobre los aportes de la fonología no lineal, Chen, Bernhardt, \& Stemberger (2016) proponen:

"By analysing all levels of the phonological hierarchy, the clinician can observe a client's strengths and needs across the phonological system from the phonological phrase to the individual feature, and provide intervention that exploits strengths at one level to address needs at another (Bernhardt \& Stemberger, 1998, 2000). This type of approach is in contrast, to, for example, phonological process analysis, with its primary focus on error patterns. Intervention studies based on nonlinear analysis and treatment methodologies have shown both short-term gains (Bernhardt, 1990; 1992; 1994; Bernhardt \& Stemberger 2000; Major \& Bernhardt, 1998; Von Bremen, 1990) and longer-term benefits (Bernhardt \& Major, 2005). Word structure gains have generalised faster than segmental gains in 
most studies, thereby enhancing intelligibility early on and setting up a strong framework for the new segments".

El conjunto de ambas unidades es utilizado en investigaciones actuales para establecer el proceso típico de adquisición fonológica y sus posibles alteraciones (Bernhardt et al., 2015; Bernhardt \& Zhao, 2010; Perez et al., 2018; Ullrich, Stemberger, \& Bernhardt, 2008); una de las cuales corresponde a una extensión de dicho proceso, más allá del tiempo esperado. Esto se conoce como Desarrollo Fonológico Prolongado (DFP), que se describe a continuación.

\section{Desarrollo Fonológico Prolongado (DFP)}

El DFP es un término propuesto por Bernhardt, Stemberger, \& Handford (1998) e inspirado en el concepto de desarrollo fonológico retardado, creado por Dodd (1995). El cambio de nomenclatura se debe a una preferencia por un concepto centrado en la normalidad y no en el déficit, pues existe la posibilidad de que los niños alcancen el desarrollo típico (DT). A partir de esto, diversas investigaciones han buscado caracterizar dicha condición a nivel lingüístico y comportamental (Bernhardt et al., 2015; Burrows \& Goldstein, 2010; Dubasik \& Ingram, 2013; Goldstein, Fabiano, \& Iglesias, 2004; Mason, 2015; Raymond, 2017).

Dubasik \& Ingram (2013) compararon el rendimiento lingüístico de seis niños monolingües hablantes de español nacidos en Estados Unidos, de entre 3.7 y 3.11 años, de los cuales tres presentaban DFP y tres DT. Los autores reportaron que los niños con DFP manifestaban patrones fonológicos típicos que persistían más allá de la edad esperada. Además, mostraron que en una interacción en contexto familiar pero con un oyente desconocido los niños con DFP tenían una inteligibilidad del habla -es decir cuan entendible es el mensaje verbal para el oyente en un contexto de comunicación- que oscilaba entre un 10 y un $25 \%$. En tanto, los niños con DT presentaban sobre un $90 \%$ en las mismas condiciones. De esta forma, pudieron establecer que los niños con DFP presentan una inteligibilidad significativamente inferior en comparación con los niños con DT.

En esta misma línea, los estudios de Burrows \& Goldstein (2010) y Raymond (2017) evaluaron el tipo de error predominante en el habla infantil de niños con DFP. El primero, analizó el rendimiento de 24 niños: ocho monolingües hablantes de español, ocho monolingües hablantes de inglés y ocho bilingües inglés-español, cuyas edades fluctuaban entre los 3.2 y los 5.3 años. El segundo, en tanto, estudió el desempeño de 59 niños granadinos: 29 con DFP y 30 control con DT, cuyas edades bordeaban entre los 3.0 y 5.0 años. Ambas investigaciones coincidieron en que el error de mayor frecuencia en el DFP fue la sustitución.

Por otra parte, en el estudio de Burrows \& Goldstein (2010) se evaluó el Porcentaje de Consonantes Correctas (PCC) en una población con DFP, el cual se define como el porcentaje de consonantes emitidas acertadamente por los niños. En dicha investigación, los autores reportaron un porcentaje de producción correcta de $65.75 \%$ para el grupo de sujetos hablantes monolingües del español.

Respecto al WSM, el estudio de Bernhardt et al. (2015) comparó 59 niños españoles (granadinos) de entre 3.0 y 5.0 años: 29 con DFP y 30 con DT, los cuales fueron divididos de acuerdo con sus rangos etarios 
(3.0 a 3.11, 4.0 a 4.11 y 5.0 a 5.11 años). Si bien existieron puntuaciones equivalentes a los cinco años, fue posible observar diferencias entre los niños del grupo control y los niños con DFP en el tramo de edad que abarcaba entre los 3.0 y 4.0 años. En este último, los niños con DT presentaron un mayor WSM en comparación con el de niños con DFP (Mann-Whitney $U=3.677, N=19, E S=0.8435, p<0.001)$,

En tanto, en el estudio de Perez et al. (2018) realizó una comparación entre 30 niños chilenos con DT y 69 niños españoles, 30 con DT y 29 con DFP, todos con una edad entre 3 y 5 años. A partir de sus hallazgos se pudo observar que los niños chilenos perteneciente al grupo de 3 años con DT tienen un mejor desempeño en los grupos consonánticos en posición inicial que el mismo grupo etario de España e incluso mejor que los sujetos de 3 y 4 años con DFP.

Mason (2015), por su parte, realizó un metaanálisis en el idioma inglés con el propósito de comparar el rendimiento de niños con DFP y el de niños con DT, tanto en edades tempranas como en preadolescentes y adolescentes; considerando aspectos como la decodificación de palabras y no palabras, la fluidez y la ortografía. Como resultado, observó que quienes estaban diagnosticados con DFP obtuvieron puntuaciones más bajas que los niños con DT; y que los niños con DT establecieron mejor la estructura de la palabra que aquellos con DFP.

Finalmente, en el ámbito conductual, los niños con DFP a menudo son percibidos como más desorganizados en comparación con sus compañeros con DT. Ellos suelen requerir intervención clínica poco tiempo después de ingresar al jardín infantil, debido a que se puede ver afectado su rendimiento académico (Dubasik \& Ingram, 2013).
Actualmente, no existen antecedentes acerca de niños chilenos de entre 3.0 y 3.11 años diagnosticados con DFP. Por este motivo, el objetivo de la presente investigación preliminar fue describir los patrones fonológicos de cinco niños chilenos de entre 3.0 y 3.11 años con DFP según el enfoque de la fonología no lineal, pertenecientes a jardines infantiles particulares subvencionados de la Región de Valparaíso, Chile.

\section{Metodología}

\section{Muestra}

En este estudio, se evaluó un total de 30 sujetos de entre 3.0 y 3.11 años, pertenecientes a diversos jardines infantiles de la Región de Valparaíso; de estos, se seleccionó a cinco niños chilenos monolingües que cumplieron los criterios de inclusión: español como lengua materna, nivel socioeconómico medio, inteligibles y con DFP. Todos los participantes fueron evaluados con pruebas estandarizadas y se excluyeron aquellos que presentaron alguna patología concomitante que pudiera explicar la alteración a nivel fonológico, tales como hipoacusias y Trastorno Específico del Lenguaje (TEL).

Para la selección de la muestra, se utilizó el muestreo no probabilístico, que correspondió a casostipo, a fin de obtener información sobre características específicas del grupo en cuestión (Hernández, Fernández, \& Baptista, 2010). Por consiguiente, la selección de los participantes dependió de los criterios de inclusión y exclusión, y no de la utilización de fórmulas probabilísticas dentro de la población escogida. 


\section{Instrumentos de selección de la muestra}

Se utilizaron diversos instrumentos para identificar el DFP en los niños que serían incluidos en la muestra, los que se presentan a continuación:

a) Audiometría: se utilizó un audiómetro Interacoustic AD226bv13. Previamente, se les realizó una otoscopía simple con otoscopio Riester E-scope HL. El niño debía tener indemnidad auditiva.

b) TEPROSIF-R: Test para evaluar los procesos de simplificación fonológica (Pavez, Maggiolo, \& Coloma, 2008). Se utilizaron las normas y criterios de corte descritos para el instrumento, rendimiento en "déficit" para el rango de edad.

c) STSG-R: test exploratorio de la gramática española (Pavez, 2003). Se utilizaron las normas y criterios de corte descritos para el instrumento, rendimiento sobre percentil 25 para el rango de edad.

d) TEVI-R: test de vocabulario en imágenes (Echeverría, Herrera, \& Segure, 2002). Se utilizaron las normas descritas por el instrumento, rendimiento en categoría "norma" o superior para el rango de edad.

e) PAE: Prueba de Articulación Eluney (Delgado

et al., 2013). Se utilizó el análisis cualitativo del instrumento.

f) Escala de Inteligibilidad en Contexto (McLeod, Harrison, \& McCormack, 2012). El niño debía tener un rendimiento con puntaje promedio mínimo de tres que corresponde "a veces ininteligible".

g) Pauta de Habilidades Pragmáticas: adaptado del Protocolo Pragmático (Prutting \& Kittchner, 1987).

h) Encuesta a padres o apoderados (Vergara, 2014). Para establecer el nivel socioeconómico y antecedentes generales. En ella se detalla: ingreso per cápita, nivel de escolaridad de los padres, lugar que ocupa el evaluado entre los hermanos, lengua utilizada con el menor y antecedentes de tratamiento fonoaudiológico.

\section{Instrumentos de evaluación de la muestra}

Para evaluar la muestra, se usó la Lista de Palabras del Español (B. Bernhardt et al., 2016). La versión anterior de esta prueba fue realizada en el año 2012 y la versión para Chile fue validada por (Vergara, 2014) mediante una muestra de niños normotípicos. La finalidad de este instrumento es representar los fonemas y las estructuras que se encuentran de manera típica en la lengua española. La versión utilizada contiene 100 palabras: 8 monosilábicas, 59 bisilábicas, 26 trisilábicas, 9 de 4 sílabas y 1 de cinco sílabas (B. Bernhardt et al., 2016). En la tabla 1, se describen las características de la Lista de Palabras del español: 
Tabla 1

Características de la Lista de Palabras del Español.

\begin{tabular}{|c|c|c|c|c|c|c|c|c|}
\hline № sílabas & Acento & Ejemplo & № palabras & \multicolumn{4}{|c|}{ Estructura Silábica } & Ejemplo \\
\hline & & & & & $\sin \mathrm{CC}$ & & Con CC & \\
\hline \multirow[t]{2}{*}{1} & $S$ & dos & 8 & $\mathrm{CV}(\mathrm{C})$ & 4 & $\operatorname{CCV}(\mathrm{C})$ & 3 & flor \\
\hline & & & & VV & 1 & & & \\
\hline \multirow[t]{4}{*}{2} & Sw & rojo & 50 & 'CV(V)(C)V(C) & 26 & 'CCVCV(V)(C) & 11 & dragón \\
\hline & & & & 'V(V)CV(V)(C) & 4 & 'CV(V)CCV(C) & 6 & cuatro \\
\hline & & & & & & 'CCVCCV & 3 & grande \\
\hline & wS & azul & 7 & (C)V'CVC & 6 & $\mathrm{CCV}^{\prime} \mathrm{CV}(\mathrm{C})$ & 1 & frutas \\
\hline \multirow[t]{4}{*}{3} & Sww & pájaro & 3 & 'CVCVCV & 2 & 'CVCCVCV & 1 & lámpara \\
\hline & wwS & pantalón & 1 & & & $\operatorname{CVCCV}^{\prime} \mathrm{CV}(\mathrm{C})$ & 1 & pantalón \\
\hline & wSw & caballo & 21 & (C)C(V)CVCV & 9 & $(\mathrm{C}) \mathrm{V}(\mathrm{C})^{\prime} \mathrm{C}(\mathrm{V}) \mathrm{C}(\mathrm{C})(\mathrm{C}) \mathrm{V}(\mathrm{C})$ & 11 & pescado \\
\hline & & & & & & $\operatorname{CCV}(\mathrm{C})^{\prime} \mathrm{CVCV}$ & 1 & princesa \\
\hline \multirow[t]{4}{*}{4} & wSww & teléfono & 1 & CV'CVCVCV & 1 & & & \\
\hline & wwSw & chocolate & 8 & CVCVCV'CV & 2 & CCVCV'CVCV & 2 & primavera \\
\hline & & & & $\mathrm{CVCV}^{\prime}(\mathrm{C}) \mathrm{V}(\mathrm{V}) \mathrm{CV}(\mathrm{V})$ & 2 & $\mathrm{VC}(\mathrm{C}) \mathrm{V}^{\prime} \mathrm{CV}(\mathrm{C}) \mathrm{CV}$ & 1 & escalera \\
\hline & & & & CVCV'CVV & 1 & & & \\
\hline 5 & wwSww & hipopótamo & 1 & VCV'CVCVCV & 1 & & & \\
\hline
\end{tabular}

Simbología: w: sílaba átona, S: sílaba tónica, V: vocal, VV: diptongo, C: consonante, CC: grupo consonántico, (): presencia opcional.

\section{Variables}

\section{Unidades suprasegmentales}

a) Whole Word Match (WWM) o Coincidencia de Palabra Completa: proporción de palabras producidas por un niño que coinciden con el modelo adulto (Bernhardt et al., 2015).

b) Word Shape Match (WSM) o Coincidencia de Estructura de la Palabra: medida que evalúa la proporción de palabras producidas por el niño que, según la estructura silábica, coinciden con el modelo adulto (Bernhardt et al., 2015).

c) Acento prosódico: rasgo fundamental que se puede definir como el mayor grado de prominencia de una sílaba con respecto a las otras dentro de una misma palabra (Real Academia Española, 2011).

\section{Unidades segmentales}

a) PCC: porcentaje de consonantes correctas producidas por los menores (Shriberg \& Kwiatkowski, 1982).

\section{Procedimiento}

La presente investigación se encuentra enmarcada en el Proyecto FONDECYT N¹1150658, cuenta con la aprobación del Comité ético-científico CEC-UV de la Facultad de Medicina de la Universidad de Valparaíso (CECO 94-15). Una vez obtenida la autorización de los apoderados por medio del consentimiento 
informado, se procedió a aplicar los instrumentos de selección de la muestra. Así, los niños y niñas que cumplieron con los criterios de inclusión fueron evaluados con la Lista de Palabras del Español mediante la técnica de cierre, se le mostraba una lámina y se le decía por ejemplo "esto es un...", el niño tenía que decir "gato" para la lámina del gato, si no respondía se realizaba imitación diferida "¿esto es un perro o un gato?", si no había respuesta, finalmente se le pedía al niño que repitiera "repite gato", obteniéndose 100 producciones verbales por cada niño. Los corpus de habla se registraron auditivamente con una grabadora de alta fidelidad Tascam DR-40 y una especialista realizó una transcripción fonética de los mismos. Las transcripciones se realizaron utilizando las normas del Alfabeto Fonético Internacional (IPA de su nombre en inglés International Phonetic Alphabet) y su fuente Doulos SIL por su compatibilidad con el software PHON 1.6 (Rose, 2004). Esta interfaz de PHON permite el análisis de los componentes fonológicos suprasegmentales y segmentales mediante la comparación de cada palabra infantil con la de un adulto nativo. Se acordó fijar un adulto como objetivo estándar para el dialecto español de Chile; también se determinaron las variantes fonéticas aceptadas en el habla adulta, para tener en cuenta dicha variación y aceptar la pronunciación del niño como correcta en caso de que no hubiera concordancia con la del adulto.

Las medidas suprasegmentales (WWM, WSM y acento) fueron operacionalizadas como 1 y 0 en una
Planilla Office Excel 2016, donde se asignó 1 para las respuestas correctas y 0 para las incorrectas; con esto, se calcularon porcentajes de producción correcta y errónea. En cuanto a la unidad segmental (PCC), se contabilizó la cantidad de consonantes correctas y se obtuvo un porcentaje relacionado con la inteligibilidad, abarcando de 0 a $100 \%$.

\section{Resultados}

En el presente apartado, se exponen los resultados obtenidos a partir del corpus de 500 palabras producidas por cinco niños (100 palabras por niño), obtenidas a partir de la Lista de Palabras del Español (B. Bernhardt et al., 2016). Esta muestra de habla fue analizada para determinar los valores de las unidades suprasegmentales (WWM, WSM y patrones acentuales) y la unidad segmental (PCC) y, de este modo, caracterizar el lenguaje del grupo en estudio. A continuación, se exponen los datos obtenidos.

\section{Unidades suprasegmentales}

\section{a) Whole Word Match o Coincidencia de la Palabra Completa (WWM)}

Se analizó la medida de WWM y se obtuvo el número total de aciertos y errores. Del mismo modo, se calculó el porcentaje de producción correcta y el porcentaje de producción errónea. En el gráfico 1, se exponen los resultados del rendimiento grupal. 


\section{WWM - Resultados a nivel de grupo}

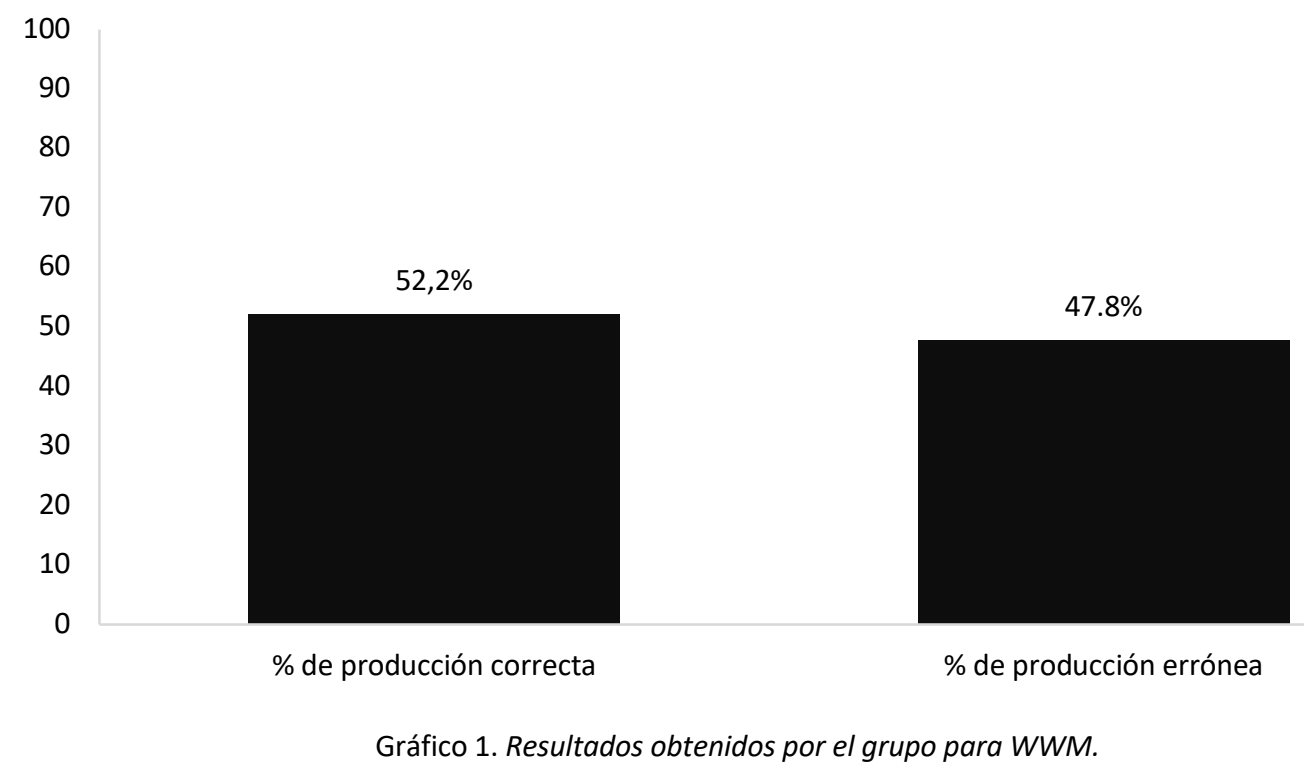

Como se puede observar en el gráfico 1, los niños produjeron un $52.2 \%$ de producción correcta, lo cual se traduce en 261 palabras emitidas igual al modelo adulto. A su vez, realizaron 239 palabras con algún tipo error, lo que corresponde a un $47.8 \%$. Un ejemplo de los errores cometidos fue la palabra [priṇ 'sẹ sa], que fue simplificada a [pin 'sẹ sa], en la que se observa una reducción del segundo elemento del grupo consonántico constituido. Otro ejemplo fue la palabra [a 'sơl], en cuyo lugar se obtiene la palabra [a 'tơl], donde se conmuta el rasgo fricativo por el oclusivo al cambiar el fonema /s/ por $/ \mathrm{t} /$.

\section{b) Word Shape Match o Coincidencia de la Estructura de la Palabra (WSM)}

Para obtener los datos de la variable WSM, al igual que en la variable anterior de WWM, se determinó el número de aciertos, el número de errores y sus respectivos porcentajes. En el gráfico 2, se exponen los resultados a nivel grupal. 


\section{WSM - Resultados a nivel de grupo}

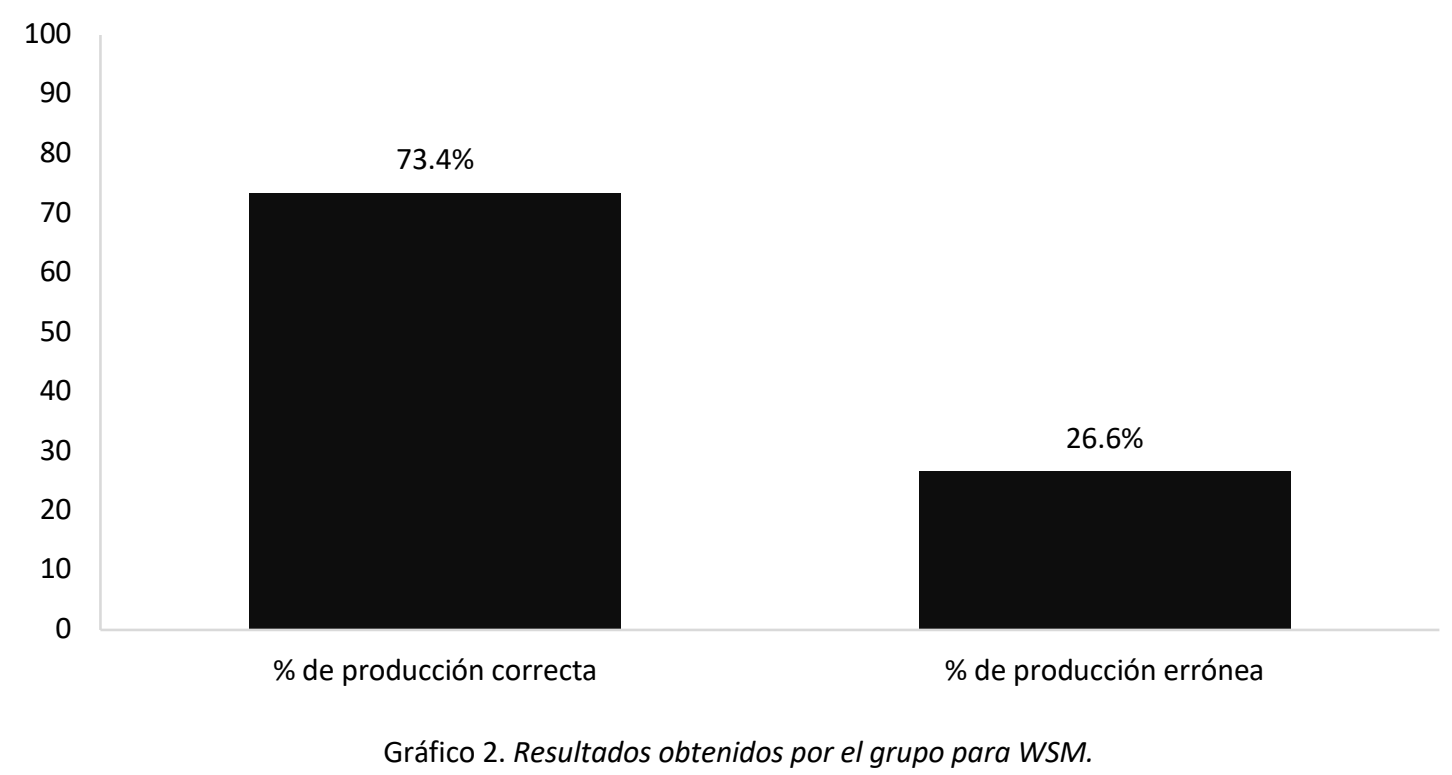

A partir de los datos expuestos en el gráfico 2 , se señalar que el porcentaje de error en WSM (26.6\%) observa un total de 367 aciertos, lo cual corresponde fue inferior al resultado en WWM (47.8\%). a un $73.4 \%$ de producción correcta a nivel de la muestra. En tanto, los participantes de la muestra presentaron un total de 133 errores, que se traduce en un $26.6 \%$ del total. Un ejemplo de los errores cometidos por los niños fue la reducción de grupo consonántico, como en el caso de [prị ma 'ßẹ ra] que fue modificado por [pị ma ' $\beta$ ẹ ra], en donde la estructura de la primera sílaba fue simplificada de CCV a CV. En cambio, la palabra ['pe rọ] fue producida como ['pẹ rọ], lo cual se consideró correcto, ya que se conservó la estructura CV, a pesar de la modificación

\section{c) Acento}

Los datos obtenidos del patrón acentual se calcularon de acuerdo con las variables anteriores. Dichos resultados se presentan en el gráfico 3. Posteriormente, en la tabla 2 , se evidencian la cantidad de errores y aciertos, además de sus respectivos porcentajes a nivel grupal, según las estructuras acentuales estudiadas en la presente investigación. en la consonante. A nivel de grupo, también se puede 


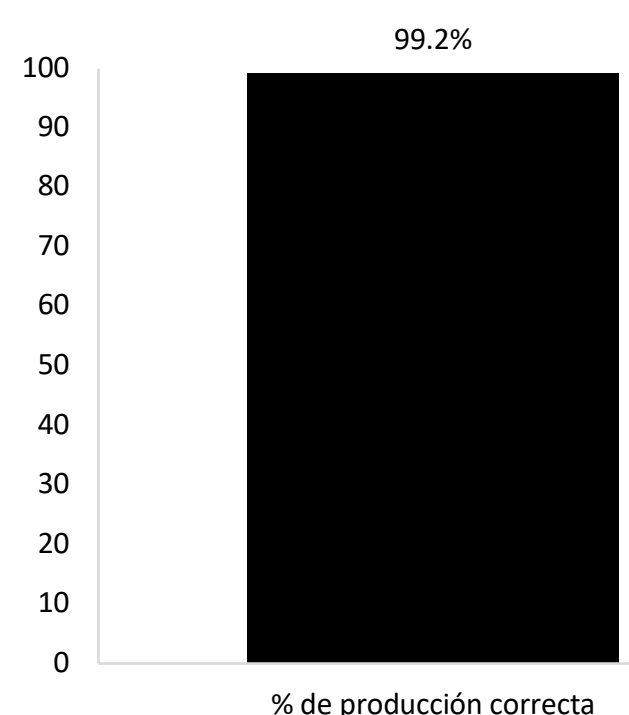

Gráfico 3. Resultados obtenidos por el grupo para los patrones acentuales.
$0.8 \%$

\% de producción errónea

\section{Acento}

Los datos expuestos en el gráfico 3 permiten observar que los niños de la muestra emitieron cuatro palabras con errores en su acentuación. Por lo tanto, su desempeño presenta un $99.2 \%$ de aciertos, muy cercano al $100 \%$; y sólo un $0.8 \%$ de error. Con esta información, se generó la tabla 2, donde se indica el número y el porcentaje de error y acierto, según los tipos de estructura acentual.

Tabla 2

Recuento de aciertos por tipo de estructura a nivel de grupo de muestra.

\begin{tabular}{|c|c|c|c|c|c|}
\hline & Total palabras & Errores & Aciertos & $\%$ Errores & $\%$ Aciertos \\
\hline$S$ & 40 & 0 & 40 & 0 & 100 \\
\hline Sw & 250 & 1 & 249 & 0.4 & 99.6 \\
\hline Sww & 20 & 0 & 20 & 0 & 100 \\
\hline wS & 35 & 0 & 35 & 0 & 100 \\
\hline wSw & 100 & 0 & 100 & 0 & 100 \\
\hline wSww & 5 & 0 & 5 & 0 & 100 \\
\hline wwS & 5 & 0 & 5 & 0 & 100 \\
\hline wwSw & 40 & 1 & 39 & 2.5 & 97.5 \\
\hline wwSww & 5 & 2 & 3 & 40 & 60 \\
\hline Total & 500 & 4 & 496 & 0.8 & 99.2 \\
\hline
\end{tabular}

Simbología. w: sílaba átona, S: sílaba tónica 
A partir de la tabla 2 se observa que, de un total de 250 palabras con patrón acentual Sw, el más frecuente en el idioma español, los niños cometieron sólo un error. En tanto, de un total de cinco palabras con el patrón acentual wwSww, de escasa frecuencia en habla hispana, se cometieron dos errores, coincidiendo con el mayor porcentaje de error en la evaluación acentual. Un ejemplo fue la producción incorrecta de la palabra [ị pọ 'pọ ta mọ], al cambiarla por [pọ 'ta mõn], donde se modifica la acentuación de esdrújula a grave. Otro error se apreció en el patrón acentual wwSw, con la palabra [kọ kọ 'đrị lọ], la cual fue producida como [kọ 'đra đrị lo], modificándose su propiedad acentual de grave a esdrújula.

\section{Unidades segmentales}

\section{a) Porcentaje de Consonantes Correctas (PCC)}

Las 1465 consonantes de la muestra fueron analizadas para calcular la totalidad de aciertos y errores, expresados en porcentajes (Porcentaje de Consonantes Correctas o PCC-C y el Porcentaje de Consonantes Erróneas o PCC-E). En el gráfico 4, se presentan los resultados para cada medida; y en el gráfico 5 , se exponen los tipos de errores cometidos a nivel grupal.

\section{PCC - Resultados a nivel de grupo}

100
90
80
70
60
5
4
30
20
10

100

90

80

70

60

50

40

30

20

10

0

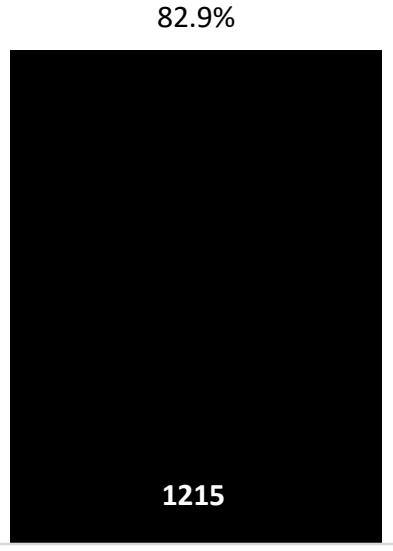

PCC-C
$17.1 \%$

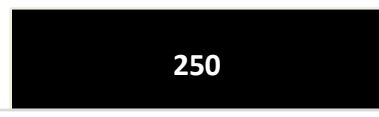

PCC-E

Gráfico 4. Resultados obtenidos por el grupo para el PCC.

En el gráfico 4, se aprecia un total de 1215 consonantes acertadas, lo que equivale a un $82.9 \%$. En contraposición, se observaron solo 250 consonantes erróneas, lo que representa un 17.1\%.
De la totalidad de errores, la sustitución fue el tipo más frecuente, representando un $57 \%$, mientras que las omisiones ocuparon un $43 \%$. Estos porcentajes se pueden observar en el gráfico 5. 


\section{PCC - Tipos de error a nivel de grupo}

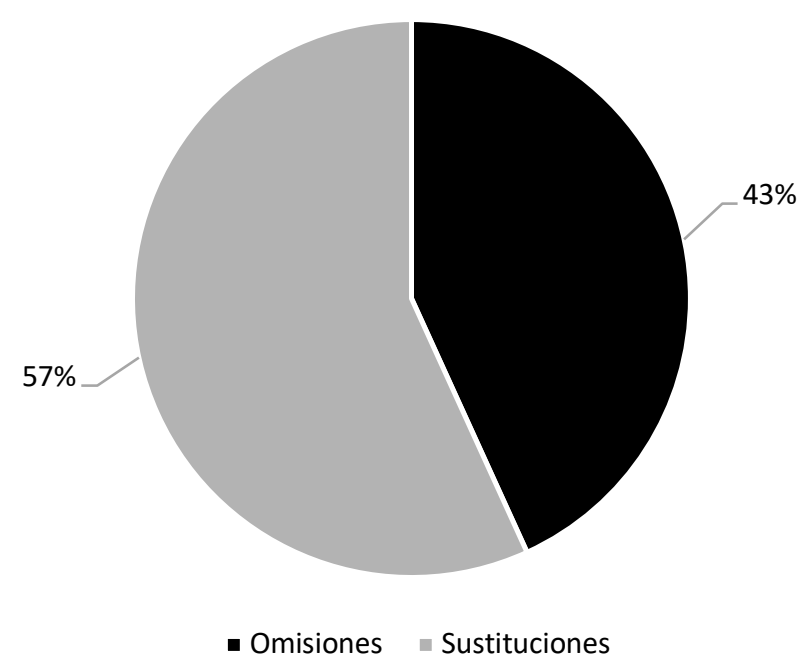

Gráfico 5. Tipos de error cometidos por los niños a nivel grupal.

Para finalizar, se analizaron los tipos de errores cometidos según las características acentuales de cada sílaba del corpus. Como resultado, se obtuvo que el $56 \%$ de errores se produjo en sílabas átonas; mientras que, un $44 \%$ en sílabas tónicas. Un ejemplo fue la producción [kọ rọ 'ra tẹ] en lugar de [t]ọ kọ 'la tẹ], donde el niño asimiló /t $/$ por /k/ y /k/ por /r/. Otro ejemplo es [ka 'lẹ ra] en lugar de [ẹs ka 'lẹ ra], en donde se omitió la sílaba /es/. En el caso de [ka 'lẹ ra], el tipo de error cometido ocurre en sílaba átona; y, en el caso de [kọ rọ 'ra tẹ], el error se produce tanto en sílaba tónica como átona.

\section{Discusión}

En relación con el WWM, los niños del presente estudio obtuvieron un $52.2 \%$ de producción correcta, mayor a la obtenida por otras investigaciones. Por ejemplo, Bernhardt et al. (2015) analizó a niños granadinos del mismo rango etario y diagnosticados con DFP, quienes presentaron un $21.4 \%$ de aciertos en este patrón suprasegmental. Este porcentaje fue inferior al obtenido por Vergara (2014) y Raymond (2017) en sus estudios sobre el desarrollo típico del lenguaje, donde observaron un $60.2 \%$ y $57.9 \%$ de aciertos, respectivamente. Por lo tanto, se puede señalar que los sujetos con DFP de la presente muestra obtuvieron un WWM inferior al de quienes tienen DT.

Respecto a la coincidencia de la estructura de la palabra (WSM), se obtuvo un $73.4 \%$ de aciertos a nivel grupal, lo cual es superior a los datos obtenidos para la variable WWM. En el estudio de Bernhardt et al. (2015) fue posible observar la misma diferencia entre WWM y WSM; No obstante, se evidenció disparidad con los datos obtenidos para la variable WSM, ya que los niños de entre 3.0 y 3.11 años de dicha investigación obtuvieron un $48.5 \%$ de aciertos. En las investigaciones de Vergara (2014) y Raymond (2017), se observa que los niños con DT presentaron un $81.5 \%$ 
y $64.7 \%$, respectivamente. En consecuencia, los porcentajes de aciertos de los niños con DFP de la muestra se encuentran descendidos en relación con los resultados de (Vergara, 2014); sin embargo, los aciertos en la producción del DFP fueron más altos que los presentados por los niños con DT del estudio de (Raymond, 2017).

En cuanto a la unidad suprasegmental relacionada con los patrones acentuales, los niños de la presente muestra obtuvieron un $99.2 \%$ de aciertos. En esta, el patrón con mayor número de palabras evaluadas fue Sw, con 50 de 100 palabras de la lista. El patrón señalado obtuvo un porcentaje de aciertos del 99.6\%, lo que corresponde a una palabra errónea. En concordancia con este resultado, Pérez (2013) encontró un rendimiento general cercano al 100\% de aciertos. Este resultado era esperable por tres motivos: la adquisición temprana del acento en los niños; la alta frecuencia de uso de la estructura Sw en la lengua española; y la definición del español como una lengua trocaica.

El valor de acierto los patrones acentuales obtenidos en esta muestra (99.2\%) es concordante con el logrado (97.7\%) por los niños con DT en el estudio de Vergara (2014) en Chile. Ello se condice con la información existente en la bibliografía, la cual establece que el patrón acentual corresponde a una de las primeras unidades suprasegmentales en ser adquiridas durante el desarrollo lingüístico. Por otro lado, el mayor porcentaje de errores acentuales cometidos se produce en el patrón wwSww, resultado coincidente con el obtenido por Vergara (2014). Al presentarse la misma falta en la palabra de tipo pentasilábica, tanto en DT como en DFP, es posible afirmar que, a mayor extensión de la palabra, mayor es la probabilidad de error en acentuación. Esto fue corroborado por Vergara (2014), quien señala que la mayor dificultad se generó por la alta metría y, además, en palabra de poco uso en el idioma.

Otro punto relevante es que en la investigación de Pérez (2013) se confirma que el patrón acentual con más errores corresponde al polisílabo wwSww o [ị pọ 'pọ ta mọ], lo que concuerda con lo observado en la presente muestra, cuyo porcentaje de error a nivel grupal fue de $40 \%$. Este resultado se explica por la elevada complejidad de la palabra y su escasa frecuencia dentro del idioma español. Además, constituye el $50 \%$ del total de errores cometidos por los niños del presente estudio en cuanto a patrones acentuales.

En la evaluación de la unidad segmental de Porcentaje de Consonantes Correctas (PCC), los niños de la muestra obtuvieron un $82.9 \%$ de aciertos y un 17.1\% de errores. De estos, 142 corresponden a sustituciones (56.8\%) y 108 a omisiones (43.2\%). Estos hallazgos coinciden con los obtenidos en la investigación de (Raymond, 2017) en una muestra de habla de 29 niños granadinos con DFP, donde también se constató que el tipo de error predominante es la sustitución de consonantes. El porcentaje de aciertos del PCC, en cambio, no concordó con lo observado en una muestra de habla de ocho hispanohablantes, realizada por Burrows \& Goldstein (2010). En esta investigación, los autores reportaron un porcentaje de producción correcta de 65.75\%, es decir, desempeño inferior al de la muestra del presente estudio.

Respecto al rendimiento de los niños con DT, la sustitución es el tipo de error más habitual en las emisiones infantiles en el grupo etario de 3.0 a 3.11 
años (Raymond, 2017; Vivar \& León, 2009). Dicha característica se conserva en el grupo con DFP muestreado en la presente investigación. Al respecto, Chávez-Peón et al. (2012) señalan que las sílabas átonas son más vulnerables de ser eliminadas en comparación con las sílabas tónicas, concordando con el puntaje obtenido en esta investigación: 139 errores en sílabas átonas y 111 en tónicas.

Para finalizar, es posible establecer que los objetivos planteados al inicio del estudio fueron cumplidos, ya que se logró describir el desempeño a nivel fonológico de niños con DFP de entre 3.0 y 3.11 años, tanto en las unidades suprasegmentales como segmentales. Además, se pudo comparar los hallazgos encontrados en el presente estudio con poblaciones de niños con DFP de investigaciones internacionales con desarrollo típico. Esto último permitió establecer semejanzas y diferencias en cuanto al DFP en Chile que, hasta ahora, no se habían realizado. Estos resultados, representan un aporte para el diseño de un plan de intervención destinado a los niños que sólo presenten alteraciones a nivel fonológico, ya que es posible contar con una base para establecer los objetivos de la terapia y adaptar el proceso terapéutico según las características individuales de cada sujeto.

\section{Conclusiones}

A partir de los resultados obtenidos, el DFP se puede describir como una condición que afecta tantos las unidades suprasegmentales como segmentales de la fonología. Esto se constata, ya que el grupo estudiado en Chile obtuvo resultados inferiores a los de los niños chilenos con Desarrollo Típico (DT) en las medidas de WWM, WSM y PCC. No obstante, se observa un patrón acentual similar a niños sin DPF, debido a que esta unidad suprasegmental es de adquisición temprana.

Las características evidenciadas en esta investigación se ven reflejadas en otras poblaciones con DFP, quienes han obtenido valores inferiores para las medidas de WWM, WSM y PCC en comparación con la población con DT. Esto se condice con lo observado y descrito en el presente estudio. Sin embargo, los niños chilenos con DFP de la muestra tuvieron un mejor desempeño en las medidas fonológicas analizadas que los niños con DFP de investigaciones internacionales (Bernhardt et al., 2015; Burrows \& Goldstein, 2010; Dubasik \& Ingram, 2013; Pérez, 2013). La escasez de estudios previos sobre el DFP en Chile limitó la presente investigación, ya que no fue posible contar con referentes de comparación para realizar un análisis cualitativo de la información.

A pesar de las limitaciones mencionadas, la presente investigación es pionera en el estudio del DFP en niños chilenos; por lo cual, sienta los cimientos para llevar a cabo nuevos estudios basados en la Teoría de la fonología no lineal. De este modo, se aporta un nuevo enfoque para el análisis y valoración de los aspectos lingüísticos de la población chilena diagnosticada con dicha condición.

En consecuencia, es posible realizar diferentes proyecciones para profundizar el conocimiento sobre el DFP. En primer lugar, sería recomendable llevar a cabo una investigación en una muestra mayor, que permita representar a la población chilena con DFP en el rango etario estudiado. En segundo lugar, sería conveniente realizar un análisis descriptivo del DFP en 
muestras de diferentes edades, con el fin de comparar los grupos etarios entre sí y observar la evolución de dicha condición a lo largo del tiempo. En tercer lugar, se debería comparar el rendimiento de niños con DFP y con DT en cada grupo etario para determinar criterios diagnósticos. En cuarto lugar, cobra relevancia el estudio comparativo de las características del DFP con otras condiciones que repercuten en el nivel fonológico del lenguaje. Finalmente, se podría ejecutar un estudio que mida la forma en que la terapia influye en las características propias del DFP, y que plantee un modelo de intervención efectivo basado en las necesidades de los niños.

Agradecimientos: esta investigación fue desarrollada en el marco del Proyecto CONICYT FONDECYT $N^{\circ} 11150658$. [This research was conducted in the context of the FONDECYT Project N 11150658].

\section{Referencias}

Bernhardt, B. H., Stemberger, J. P., \& Handford, B. (1998). Handbook of Phonological Development from the Perspective of Constraint-based Nonlinear Phonology. Academic Press.

Bernhardt, B., Hanson, R., Perez, D., Ávila, C., Lleó, C., Stemberger, J. P., ... Chávez-Peón, M. (2015). Word structures of Granada Spanish-speaking preschoolers with typical versus protracted phonological development. International Journal of Language \& Communication Disorders, 50(3), 298-311. https://doi.org/10.1111/14606984.12133

Bernhardt, B., Mendoza, E., Carballo, G., Pérez, D., Ávila, C., Fresneda, D., ... Stemberger, J. P. (2016). Prueba de Fonología en Español (100 palabras). Recuperado de http://phonodevelopment.sites.olt.ubc.ca/espanol_prueba -de-fonologia-100-palabras_2016_bmbetal_a4_reduced/

Bernhardt, M., \& Zhao, J. (2010). Nonlinear phonological analysis in assessment of protracted phonological development in Mandarin. 34(3), 168-180.

Bloomfield, L. (1984). Language. University of Chicago Press.

Burrows, L., \& Goldstein, B. A. (2010). Whole word measures in bilingual children with speech sound disorders. Clinical Linguistics \& Phonetics, 24(4-5), 357-368. https://doi.org/10.3109/02699200903581067

Chávez-Peón, M. E., Bernhardt, B. M., Adler-Bock, M., Avila, C., Carballo, G., Fresneda, D., ... Stemberger, J. P. (2012). A Spanish pilot investigation for a crosslinguistic study in protracted phonological development. Clinical Linguistics \& Phonetics, 26(3), 255-272. https://doi.org/10.3109/02699206.2011.608463

Chen, R., Bernhardt, B., \& Stemberger, J. (2016). Phonological assessment and analysis tools for Tagalog: Preliminary development. Clinical Linguistics \& Phonetics, 30(8), 599-627. https://doi.org/10.3109/02699206.2016.1157208

Chomsky, N., \& Halle, M. (1979). Principios de fonología generativa. España: Fundamentos.

Clements, G. (1985). The Geometry of Phonology Features. En C. Ewen \& J. Anderson (Eds.), Phonology Yearbook (Vol. 2, pp. 225-252). Recuperado de http://www.jstor.org/stable/4419958

Delgado, L., Figueroa, A., Martínez, C., Pereira, M., Rojas, N., \& Vergara, C. (2013). Creación de una Prueba de Articulación de fonemas para niños y niñas de 3 años a 5 años 11 meses, del Colegio Raúl Silva Henríquez y del Jardín Infantil Brotes Nuevos, de la comuna de Viña del mar, Región de Valparaíso. Universidad de Valparaíso, Valparaíso, Chile.

Dodd, B. (1995). Differential Diagnosis and Treatment of Children with Speech Disorder. London: Whurr.

Dubasik, V. L., \& Ingram, D. (2013). Comparing phonology of dyads of children with typical development and protracted development. Clinical Linguistics \& Phonetics, 27(9), 705719. https://doi.org/10.3109/02699206.2013.800134

Echeverría, M., Herrera, M., \& Segure, J. (2002). Test de Vocabulario en imágenes (TEVI-R). Recuperado de https://sites.google.com/site/zamornogomez/baterias-deevaluacion/test-de-vocabulario-en-imagenes-tevi

Goldsmith, J. (1976). Autosegmental phonology 
(Massachusetts Institute of Technology). Recuperado de http://www.ai.mit.edu/projects/dm/theses/goldsmith76.p df

Goldstein, B., Fabiano, L., \& Iglesias, A. (2004). Spontaneous and imitated productions in Spanish-speaking children with phonological disorders. Language, Speech, and Hearing Services in Schools, 35(1), 5-15.

Harris, Z. (1951). Methods in Structural Linguistics. Chicago: University of Chicago Press.

Hernández, R., Fernández, C., \& Baptista, M. del P. (2010). Metodología de la Investigación ( $5^{\mathrm{a}}$ ed.). Recuperado de https://www.esup.edu.pe/descargas/dep_investigacion/M etodologia\%20de\%20la\%20investigaci\%C3\%B3n\%205ta\%2 0Edici\%C3\%B3n.pdf

Hooper, J. B. (1976). An introduction to natural generative phonology. Academic Press.

Ingram, D. (1983). Trastornos fonológicos en el niño. Barcelona: Médica y Técnica.

Jones, D. (1957). The History and Meaning of the Term 'Phoneme'. En E. Fudge (Ed.), Phonology: Selected Readings (pp. 17-34). Harmondsworth: Penguin.

Kager, R. (1990). Strict Binary and Destressing Rules (unpublished). EE.UU.: Stanford University.

Kiparsky, P. (1982). Lexical morphology and phonology. En Linguistics in the morning calm (pp. 3-91). Seoul: Hanshin.

Liberman, M. (1975). The Intonational System of English (Massachusetts Institute of Technology). Recuperado de http://www.ai.mit.edu/projects/dm/theses/liberman75.pd $f$

Liberman, M., \& Prince, A. (1977). On Stress and Linguistic Rhythm. Linguistic Inquiry, 8, 249-336.

Lloret, M.-R. (1992). Les africades i la representació fonològica no-lineal: Estructures de contorn. Marges, Els: revista de llengua $i$ literatura, 47-63. Recuperado de https://www.raco.cat/index.php/Marges/article/view/111 358

Mason, G. (2015). Multisyllabic word production of schoolaged children with and without protracted phonological development (University of British Columbia). https://doi.org/10.14288/1.0221497

McCarthy, J. J., \& Prince, A. (1993). Prosodic Morphology I:
Constraint Interaction and Satisfaction. Rutgers Center for Cognitive Science, Rutgers, the State University of New Jersey.

McLeod, S., Harrison, L., \& McCormack, J. (2012). Intelligibility in Context Scale Bathurst. Recuperado de https://cdn.csu.edu.au/_data/assets/pdf_file/0010/3999 70/ICS-English.pdf

Mohanan, K. (1982). Lexical Phonology. University Linguistics Club, Indiana.

Pavez, M. M. (2003). Test exploratorio de gramática española de A. Toronto. Aplicación en Chile. Recuperado de http://ediciones.uc.cl/ediciones-uc-educaci\%C3\%B3n-testexploratorio-gramatica-espanola-toronto-p-468.html

Pavez, M. M., Maggiolo, M., \& Coloma, C. J. (2008). Test para evaluar procesos de simplificación fonológica: TEPROSIF-R. Santiago, Chile: Ediciones UC.

Pérez, D. (2013). Descripción de la fonología en niños con desarrollo fonológico prolongado, trastorno del desarrollo del lenguaje y síndrome de down (Http://purl.org/dc/dcmitype/Text, Universidad de Granada). Recuperado de https://dialnet.unirioja.es/servlet/tesis?codigo $=58247$

Perez, D., Vivar, P., Bernhardt, B. M., Mendoza, E., Ávila, C., Carballo, G., ... Vergara, P. (2018). Word-initial rhotic clusters in Spanish-speaking preschoolers in Chile and Granada, Spain. Clinical Linguistics \& Phonetics, 32(5-6), 481-505.

https://doi.org/10.1080/02699206.2017.1359852

Prieto, P. (2014). Introducción: La fonología. En R. Núñez, S. Colina, \& T. Bradley (Eds.), Fonología Generativa Contemporánea de la Lengua Española, segunda edición. (2 ed.). EE.UU.: Georgetown University Press.

Prutting, C., \& Kittchner, D. (1987). A Clinical Appraisal of the Pragmatic Aspects of Language. Journal of Speech and Hearing Disorders, 52(2), 105-119. https://doi.org/10.1044/jshd.5202.105

Raymond, G. (2017). Consonant acquisition in typically developing preschool speakers of Granada Spanish. University of British Columbia, Vancouver, Canadá.

Real Academia Española. (2011). Nueva gramática de la lengua española. Barcelona, España: Espasa Libros, S. L. U.

Rello, L. (2007). La relación entre Fonética y Fonología. 
https://www.academia.edu/2677821/La_Relaci\%C3\%B3n_ entre_Fon\%C3\%A9tica_Y_Fonolog\%C3\%ADa

Rose, Y. (2004). Phon (Versión 1.6). Recuperado de https://phon.ca

Shriberg, L. D., \& Kwiatkowski, J. (1982). Phonological disorders III: A procedure for assessing severity of involvement. The Journal of Speech and Hearing Disorders, $47(3), 256-270$.

Stampe, D. (1969). The acquisition of phonetic representation. 433-444. Chicago: Linguistic Society.

Ullrich, A., Stemberger, J. P., \& Bernhardt, B. M. (2008). Variability in a German-Speaking Child as Viewed from a Constraint-Based Nonlinear Phonology Perspective. Asia Pacific Journal of Speech, Language and Hearing, 11(4), 221-237. https://doi.org/10.1179/136132808805335536

Vennemann, T. (1971). Phonological Uniqueness in Natural Generative Grammar. Glossa, 6, 105-116.

Vergara, P. (2014). Patrones fonológicos en niños entre 3.0 y 5.11 años con desarrollo típico del lenguaje según el enfoque de la fonología no lineal y fonología natural que asisten a jardines infantiles de Puerto Montt. Universidad de Valparaíso, Viña del Mar, Chile.

Vivar, P., \& León, H. (2009). Desarrollo fonológico-fonético en un grupo de niños entre 3 y 5, 11 años. Revista CEFAC, 11(2), 190-198. https://doi.org/10.1590/S151618462009000200003 